\title{
EL DEPARTAMENTO DE GRADUADOS Y DE INTERNADO DE LA FACULTAD DE MEDICINA
}

Prof. O. Garcia Rosell

La Facultad de Medicina creó, a propuesta del Decano y por acuerdo unánime de la Junta de Catedráticos del 24 de Enero de 1957, el Departamento para la Enseñanza de Graduados y de Internado, dando así un paso trascendente en el desarrollo del programa que, para la reforma efectiva de los estudios médicos, está llevando a cabo. El viejo decir que afirma que el Médico es el estudiante eterno, es la expresión de una verdad que se cumplía por simple esfuerzo individual cuando las fronteras de los conocimientos parecían accesibles o cercanas. La magnitud y velocidad de las nuevas aportaciones de la investigación, hace hoy difícil que el simple esfuerzo personal, aislado, pueda mantener una información constante de tal naturaleza que permita a cada profesional la renovación permanente de sus conocimientos. De otro lado, el objetivo primero e inmediato de las escuelas de medicina, es el de la formación de médicos generales con conocimientos básicos y capacidad de ejercicio inmediato. Esta finalidad es más precisa en los países cuyo desarrollo general está en la cundición del nuestro, en los que la mayor parte de su territorio, fuera de las ciudades grandes, tiene escasos recursos asistenciales. De otro lado, el ejercicio mismo confirma la realidad ya prevista desde los estudios en la Escuela, de que la preparación básica con ser fundamental y decisiva, no puede abarcar la enorme extensión de los conocimientos que día a día enriquecen la Ciencia Médica, tanto en lo especulativo como en lo que demanda aplicación práctica inmediata. El enciclopedismo que fué concepto filosófico del siglo XVIII no es aplicable ya en la cultura general y está fuera de toda posible consideración, aun dentro de una acomodada restricción a las actividaeds humanas en la ciencia o en la técnica. El 
nacimiento de las especialidades no ha sido fruto de un deseo caprichoso para restringir el campo del trabajo; por el contrario, aquellas han nacido de la necesidad que siente el médico de ser más seguro en su capacidad y más eficaz en su ejercicio. La focalización de sus estudios evita, de otra manera, la dañosa dispersión. Limitando el campo se adquiere mayor profundidad. La reacción en general del paciente es regular y uniforme frente a este sentido de especialización. Cada quien, en el ambiente laico, liene un concepto preciso de que las alteraciones de su salud, si bien tienen manifestaciones que conducen $a$ un desequilibrio general, se sistematizan en forma que llaman a una atención más focalizada. Espontáneamente, el público busca el auxilio de los especialistas. El Médico General, es el confidente inmediato, es el ser en quien pone su confianza de primera intención y la mantiene en tanto sus males o son atenuados o no momifiestan un carácter muy específico. Es, también, quien le cuida y le guía en cuanto a la necesidad de atención especializada. Pero, si bien las especialidades nacieron de la necesidad y crecieron como resultado inmediato de la exiensión de la ciencia y del descubrimiento de hechos nuevos en cuanto al origen y patogenia de las enfermedades, en tanto que aumentaron los recursos auxiliares de diagnóstico, terapéutica y prevención, es verdad también que en su aparición y crecimiento no ha habido un orden definido. La formación del especialista se ha hecho por inclinación espontánea, por búsqueda individual de campo de trabajo o por simpatía especial a determinado profesor. Con frecuencia se solicita la formación de más médicos generales para cubrir las necesidades de nuestra población comprendida en un complejo racial, cultural y económica dentro de la aun más compleja contormación topográfica de nuestro territorio. Poco podrán hacer los médicos generales, aislados en lugares sin recursos asistenciales y sanitarios. Cuanto mejor haya sido su formación en la escuela médica, sentirá más urgentemente, más dolorosamente podríamos decir, la falta del colega especializado, patólogo, radiólogo, microbiólogo, pediatra, obstetra, etc., etc., y cuanto más urgirá la colaboración del sanitarista. La solución del problema no vendrá solamente en la formación de más y mejores médicos generales, sino, además, en la preparación de más y mejores especialistas para formar los equipos de trabajo. No habiendo sistematización. la formación actual de especialista está falta, generalmente, de la preparación en las ciencias básicas sobre las que asienta tojo posible desarrollo de la enfermedad. Siendo un adiestramiento de aplicaciones diarias, adolece de la falta de discusión de la doctrina en que se funda aquella. Además, con frecuen- 
cia se parcializa en la rutina de técnica, más que en el juicio científico.

Siendo la Medicina, ciencia y profesión de génesis; desarrollo y xplicación mundial, la necesidad de mantener un sistema de relación entre las Escuelas y los Graduados, para continuar la educación e instrucción organizadas, esto es, para fortalecer con organismos fehacien. tes el vínculo espiritual entre los prrofesionales. y los ceniros docentes y de investigación, ha sido sentida uniformemente y en cada país se ha buscado la solución mejor acomodada a sus realidades y necesidades nacionales. En donde la enseñanza de la Medicina está a cargo, principalmente de entidades privadas, aunque bajo normas o supervisión de organismos oficiales, la enseñanza post-grado sigue las mismas bases de sustentación. Entre nosotros la Educación Médica es oficial, a cargo de Universidades sobre las que el Estado ha delegado su autoridad y por lo tanto, es ratural que ellas tengan la responsabilidad de la organización y control de la enseñanza para Graduados y mantengan la autoridad para hacer las calificaciones de quienes se acojan a los beneficios de esta instrucción.

Al habérsenos confiado la organización del departamento, nos hemos hecho cargo de la responsabilidad que ello significa y hemos procurado valernos de la experiencia nacional, así como de las informaciones que sobre este trabajo es hecho por otros países; tratando en todo caso de comparar las experiencias ajenas, en función de las diferencias ambientales.

En nuestro cuadro general de trabajo existen dos campos vecinos, pero diferentes: 1) el del internado y 2) la enseñanza post-grado.

El Internado.- Entre nosotros, el Internado constituye el período obligatorio de transición entre la aprobación del curriculum y las pruebas para optar el Título de Médico Cirujano que autoriza el ejercicio libre de la profesión. Existió siempre en nuestra organización. En los primeros tiempos, cuando el número reducido de alumnos se adaptaba holgadamente a los servicios hospitalarios, y el número de Médicos de hospital era escaso, el Interno se agregaba por inchinación o simpatía a un Servicio, haciendo en el, él mismo, gran parte de la práctica hoy encargada al personal de enfermería; recibiendo por trato directo con el Jefe del Servicio las indicaciones derivadas. de aquella clínica intuitiva y aquella terapéutica razonada sobre la observación de casos análogos. Los servicios cuyos Jefes eran más famosos, eran más solicitados y muchos otros quedaban vacantes. Una innovación interesante fué el establecimiento del concurso para el internado. Al terminar el sexto año, se rendían pruebas de Medicina y Cirugía para obtener un diploma 


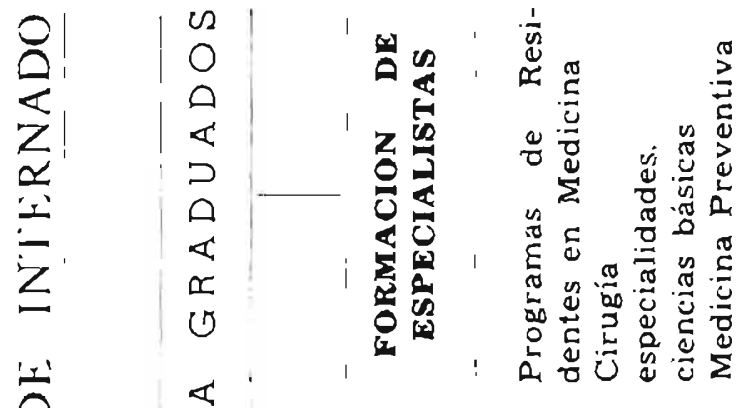

穴,

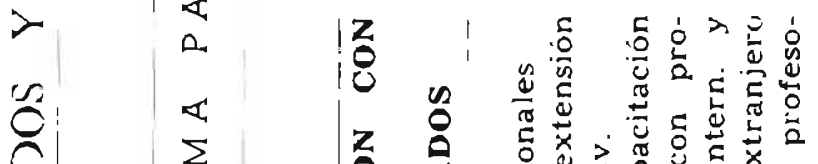

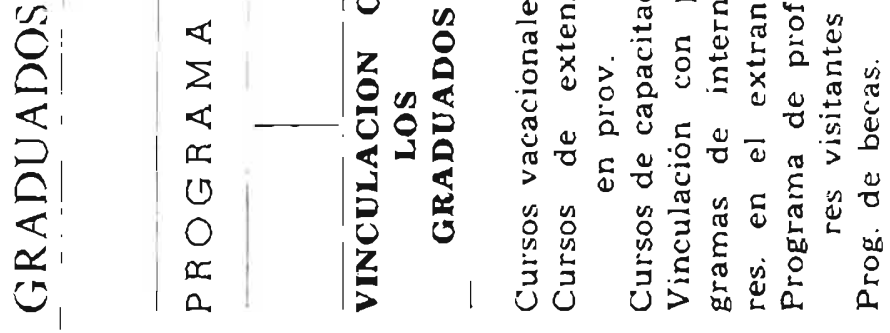

$\frac{\alpha}{\alpha}$

a.

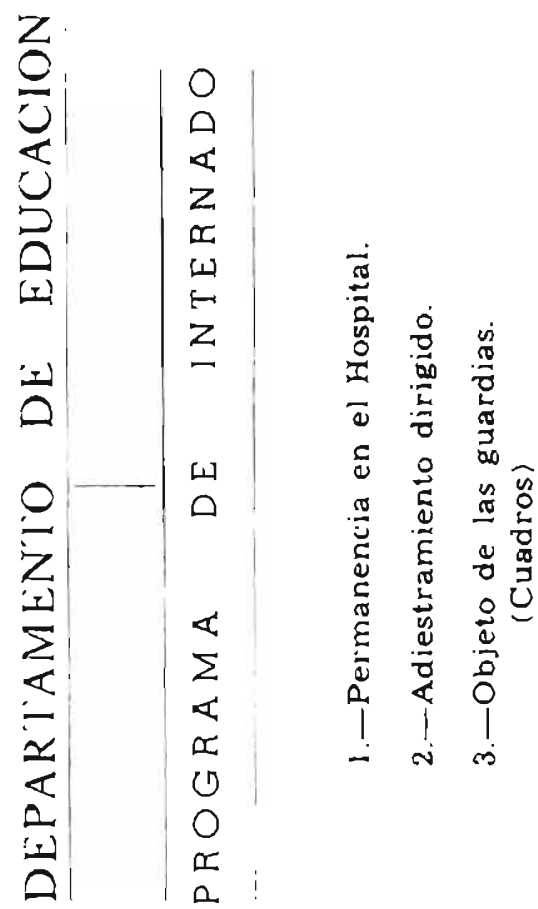


que, a su vez, era un prerequisito para postular el Grado de Bachiller. El Inlernado no era rotatorio y con frecuencia, se hacia en un solo servicio, durante todo el año. El crecimiento del curriculum había invadido el sétimo año y el Interno debía concurrir a clases en diversos lugares. Su función se limitabá a scompañar la visita de rutina en las mañanas, hacer las historias de los casos nuevos y hacer una guardia uni o bipersonal cada diez o quince días. La tercera innovación importante fué la de la supresión del requisito de concurso y la adopción del sistema actual del internado roialorio, complementado ulteriormente con el paso de los cursos regulares al. programa de años inferiores, con el saludable propósito de que los alumnos dediquen el día entero a su permonencia en el hospital. Este programa ha sido frustado por la invasión dé promociones que han sobrepasado en número al de todas las posibilidades de enseñanza regular dentro de la Facultad. Y porque el alumnado, en buena proporción, no aprovecha este tiempo para permanecer en el Hospital, sino para buscar ocupaciones remunerativas en otros ambientes. Las autoridades médicas de los hospitales, como los Profesores y los propios alumnos, han hecho crítica a este sistema que puede condensarse en los siguientes puntos: a) la permanencia de los internos en los servicios está reducida a parte de las mañanas, ignorando lo que ocurre en el resto del día; b) las quardias son irregulares y se refieren a atenciones de emergencia; y c) no hay instrucción, dirección o control del Internado. En estas condiciones se ha llegado al período actual.

El Depariamento ha puesto en marcha, desde el comienzo del presente ciclo un programa tendiente a remediar esa situación. Los fundamentos de ese programa son: a) permanencia del interno durante las horas de la mañana y de la tarde en el hospital; b) programa de instrucción sobre materias de aplicación inmediata a cargo de instructores ad-hoc; c) ciclos de prácticas con pacientes externos. El programa desarrollado corresponde a los siguientes cuadros:

\section{Cuadro $N^{\circ} 1$}

MEDICINA ( 4 meses)

8 a 9 a.m. Trabajo en consultorio externo.

9 a.m. a 12 m. Visita y trabajo en las salas bajo la dirección del personal médico. En los servicios en ios que el personal médi- 
dico asistencial no permanezca en las salas, tendrán la vigilancia de un Instructor.

2.30 a 4 p.m.-- Historia de los casos nuevos. Realización de los exámenes inmediatos. Formulación de los pedidos de análisis y consultas de las emergencias de las salas con el médico residente.

4 a 6 p.m.-Práctica a cargo de instructores según el cuadro siguiente:

2 Semanas.-Prácticas de técnicas de laboratorio e interpretación de análisis. Especial énfasis a los de emergencia.

1 semana.-Ejercicio de Interpretación de imágenes radiólogicas.

l Semana.-- Problemas epidemiológicos y sanitarios conexos a los casos de clínica.

2 Semanas.-Ejercicio de Anatomía Patológica. Interpretación de macro y micro especímenes.

2 Semanas.-Interpretación de Electrocardiogramas. Emergencias en enfermedades cardiocirculatorias.

8 Semanas.-Presentación y discusión de casos clínicos. Problemas clínicos de emergencia. Problemas terapéuticos con especial referencia a casos actuales.

\section{EN LAS GUARDIAS}

En las mañanas.- Trabajo en las salas en que están asignados.

12 m. a 2 p.m.- Almuerzo y descanso, salvo emergencias.

2 a 6 p.m.- Ayuda al Residente y asistencia a los programas de instrucción.

6 a 8 p.m.-Comida y descanso salvo emergencias.

8 p.m. a 8 a.m.-Los internos se distribuirán en tres grupos de manera que corresponda: cuatro horas de permanencia en la sala de emergencia, cuatro de ronda en las salas atendiendo el post-operatorio y cuatro en reposo en el Departamento de Internos.

\section{Cuadro No 2}

\section{CIRUGIA $(4$ meses}

8 a.m. a 12 m.- Trabajo en las salas bajo la dirección del personal médico. Visita, curaciones, Ayudantía en Anestesia y en 
las intervenciones quirúrgicas según organización interna del servicio y rol de rotación.

2.30 a 4 p.m.- Historia de casos nuevos. Exámenes inmediatos. Formulación de pedidos de análisis y medicación inmediata con revisión del Residente.

4a 6 p.m.-Práctica a cargo de Instructores según el cuadro siguiente: 4 Semanas.-Técnica operatoria en cadáveres y animales.

4 Semanas-Cirugía de Emergencia (pequeña y mayor).

2 Semanas.-Anestesia, pruebas funcionales y cuidados pre y postoperatorios.

4 Semanas.-Presentación de casos. Discusión diagnóstica.

\section{EN LASS GUARDIAS}

En las mañanas. - Trabajo en las salas en que están asignados.

12 m. a 2 p.m.-Almuerzo y descanso, salvo emergencias.

2 a 6 p.m.- Ayuda al Residente y asistencia a los programas de Instrucción.

6 a 8 p.m.-Comida y deșcanso salvo emergencias.

8 p.m. a 8 a.m.-Los internos se distribuirán en tres grupos de manera que corresponda: cuatro horas de permomencia en la sala de emergencia, cuatro de ronda en las salas atendien. do al post-operatorio y cuatro en reposo en el Departamento de Internos.

Cuadro No 3

OBSTETRICIA (2 Meses)

8 a 9 a.m.-Atención prenatal e nel consultorio, con instructores.

9 a $12 \mathrm{~m}$.- Atención en las salas de puerperio. Ayudantía en las intervenciones. Pequeñas intervenciones con autorización y vigilancia del personal médico.

2.30 a4 p.m.- Asistirán al desarrollo de los programas de instrucción en la forma siguiente:

2 Semanas.-- Emergencia en clínica obstétrica.

1 Semana-El Laboratorio en el control del embarazo, parto y puerperio. 
2 Semanas.-- Discusión Anatomo-Patológica de casos: Madre-Niño.

2 Semanas. Discusión de casos clínicos.

1 Semana.-Responsabilidad Médica en el control del embarazo.

\section{SALA DE TRABAIO}

4 a 6 p.m.- Práctica esn las salas de trabajo de sus respectivos servicios. Durante el trabajo de parto y el período expulsivo, controlarán el estado general de las pacientes y horán práctica obstétrica en rol con las alumnas, en colaboración con la matrona del Servicio. Notificarán inmediatatamente al residente de guardia en casos de complicaciones. Con instructores.

\section{EN LAS GUARDIAS}

En las mañanas los internos atenderán su propio servicio.

12 m. a 2.30 p.m.- Almuerzo y reposo salvo emergencias.

2.30 a 3 p.m.- Permanecerán en el servicio de Admisión e iniciarán las historias clínicas de los ingresos. Notificarán al Residente de todo ingreso de emergencia.

4 a 6 p.m.- Asistirám a los ejercicios de Instrucción, estando atentos a las emergencias.

6 a 8 p.m.-Comida, descanso, salvo emergencias.

8 p.m. a 8 a.m.-- Se distribuirán en tres grupos de manera que uno permanezca en admisión durante cuatro horas, otro en las salas de trabajo y el tercero en el Depto. de guardia para asistir al Residente en las intervenciones de emergencia.

\section{Cuadro No 4}

\section{PEDIATRIA (2 Meses)}

Lunes: 2.30 a 4 p.m.-Pacientes de Consultorio externo.

4 p.m. a 6 p.m.- Anatomía Patológica.

Martes: 2.30 a 4 p.m.-Pacientes de Consultorio.

4 p.m. a 6 p.m.- Terapéutica.

Miércoles: 2.30 a 4 p.m.-Pacientes de consultorio externo.

4 p.m. a 6 p.m.-Dietética.

Jueves: 2.30 a 4 p.m.- Laboratorio. 
4 p.m. a 6 p.m.-Casos quirúrgicos.

Viernes: 2.30 a 4 p.m.-Casos clínicos. Emergencia.

4 p.m. a 6 p.m.- Pacientes de consultorio externo.

Sábado: 2.30 p.m. a 4 p.m.-Casos clínicos.

4 p.m. a 6 p.m.-Interpretación de Radiografías.

\section{EN LAS GUARDIAS}

En las mañanas trabajo en los servicios asignados.

12 m. a 2 p.m.-Almuerzo y descanso.

2 a 4 p.m.- Permanencia en emergencias.

4 a 6 p.m.- Asistencia a los programas de instrucción.

6 a 8 p.m.- Comida y descanso salvo emergencias.

8 p.m. a 8 a.m. - Se distribuirán en tres grupos de manera que cada cuatro horas corresponda a cada uno, permanencia en admisión, ronda en las salas y reposo.

La realización de este programa ha demandado un esfuerzo considerable. Se ha tenido la suerte de encontrar la colaboración de número de instructores altamente eficientes y de buena voluntad, dentro del profesorado joven, quienes con la experiencia previa y la re novada en este año, harán posible el perfeccionamiento del programa para el futuro.

La experiencia del año en curso, nos ha confirmado una vez más, la imposibilidad de realizar cualquier reforma con promociones numerosas. Más de quinientos internos que han hecho los seis años anteriores en las mismas condiciones de piétora se adaptan dificilmente a una nueva disciplina. Muchos de ellos confiados en la laxitud de las obligaciones en años anteriores, han contraído compromísos extrauniversitarios, que les obligan a dedicar gran parte del tiempo a trabajos que no tienen nada que hacer con la Medicina. Otros han optado por colocaciones que, si bien estám relacionadas con aquella, no corresponden al sentido natural y propio del internado. Todo esto concurre a manifestarse en ousentismo.

Apesar de estos factores negativos, justo es declarar que el grupo de internos que ha concurrido regularmente a sus nuevas obligaciones ha recibido un beneficio que compensa el esfuerzo realizado por la Facultad. 
Tanto al iniciarse el programa, como en reuniones especiales con los grupos de cada uno de los hospitales, se ha hecho explicación del sentido de cada una de las partes componentes del programa de insirucción; tales como: la importancia del trabajo diario de una hora en consultorios externos, en donde se estudia una clínica diferente a la que se encuentra en los hospitalizados, pero similar a la que habrán de encontrar en sus consultas privadas cuando estén en el ejercicio profesional; la importarcia de aprender técnicas y ejercitar interpretaciones con especial énfasis a casos de emergencia; la correlación de la Anatomía Patológica a la clínica diaria; la renovación de las prácticas de cirugía experimental; la práctica de cuidados pre y postoperatorios, etc., etc., etc: Especial mención se hizo de la incorporación de un corto ciclo sobre problemas epidemiológicos conexos a la clínica.

Para el desarrollo del programa ha sido necesaria la adquisición de implementos de trabajo y en algunos casos, crear departamentos, tales como el dedicado a cirugía experimental en el Hospital Dos de Mayo para realizar función similar a la del ya existente en el Hospital Loayza.

Con el objeto de que la práctica de laboratorio de los internos sea más efectiva y de aplicación más inmediata, la Facultad ha dispuesto la construcción de dos edificios que serán equipados para laboratorios, uno en el Hospital Loayza y otro en el Dos de Mayo, destinados exclusivamente a la práctica de análisis de emergencia ejecutados por los propios internos y en donde puedan seguir, en determinados casos, las pruebas de laboratorio de sus pacientes. Los planos han sido ya aprobados y se cuenta con la autorización de la Sociedad de Beneficencia Pública para utilizar el terreno respectivo. Solamente el costo aproximado de las edificaciones está calculado en S/. 700,000.00. Entrarán en función en el programa de 1958.

El número de internos para el año próximo, será aproximadamente de 350, lo cual hará factible la intensificación de las aplicaciones prácticas y el control más eficaz de la permanencia hospitalaria en la mayor parte del día y la aplicación mejor del verdadero sentido de las guardias, las que no deben limitarse a atenciones de emergencia, sino a trabajo continuado para la marcha regular de los servicios. En 1959 con un número menor aun, de 200, eștaremos. más cerca de las condiciones ideales. 


\section{EL PROGRAMA DE ENSEÑANZA PARA GRADUADOS}

La doble preocupación en la enseñanza para Graduados, la del mejoramiento constante de la preparación de los Médicos Generales y la de la formación de Especialistas ha sido considerada en el programa actualmente en marcha.

Sobre la primera se han realizado las siguientes actividades: El programa de cursos vacacionales realizado en el mes de Marzo último comprendió veinte cursillos sobre materias de ciencias básicas, grandes clínicas y especialidades. Tomaron parte activa en la enseñanza 142 miembros del personal docente de la Facultad, incluyendo once Profesores Principales Titulares y 32 Profesores Principales Asociados, siendo el resto Jefes de Clínica, de Laboratorio y Ayudantes, cuya eficiencia quedó demostrada una vez más. Concurrieron 288 médicos inscritos regularmente, de los cuales 38 fueron provenientes de Provincias y 250 de Lima. Además, a las conferencias, en determinados capítulos, asistió un número considerable de colegas no inscritos. Una revisión de los programas de esos cursos hace notar los siguientes hechos significativos: Ha habido este año mayor interés en los graduados sobre temas quie importan revisión de ciencias básicas; ha habido mayor tendencia en el profesorado a abondonar la conferencia nosográfica para incluir capítulos de más reciente renovación. En los cursos de especialidades se ha notado el doble interés en docentes y graduados de difundir y conocer las nuevas adquisiciones que, siendo especializadas, deben modificar el criterio actual del internista, de un lado, y la de informar a los propios especialistas del balance de las nuevas adquisiciones en sus respectivos campos.

En general, el espíritu de estos cursillos, que es el de dar una oportunidad para revisar en forma sintética nuevas informaciones $y$ con ello despertar el interés de los graduados para la búsqueda de mayor información sobre los puntos que le hayan provocado más inquietud, fué bien cumplido. Una manifestación bien expresiva de esto es el hecho de que a la terminación del ciclo, hubo mayor número de solicitudes de graduados para agregarse voluntariamente al trabajo de las Cátedras que participaron en aquel.

Diferente al tipo de cursos vacacionales es el que se desarrolla durante el año normal. Estos tienen más carácter de actualización y con más énfasis en la práctica. A este tipo han correspondido los cursos dictados por el Prolesor Jorge Valdeavellano y el personal de su Cátedra en Oftalmología, durante el mes de octubre, con dos secciones. 
una sobre fondo de ojo para médicos generales y otra para especialistas; y el de capacitación en Urologia, organizado por el Profesor Alejandro Higginson. Dado el carácter eminentemente práctico de esta enseñanza, que mantiene el contacto directo del profesor y el graduado, el número de inscritos es limitado.

Con el propósito de hacer más regular esta clase de enseñanza se está preparando el programa para el año 1958. En tal sentido se ha solicitado a las Cátedras la confección del programa correspondiente a sus actividades, sugiriéndoles que incluyan, por lo menos, un curso corto de vacaciones, para $\in \mathrm{l}$ mes de Marzo y otro más extenso, sobre un capítulo definido. Cada uno de esos programas deberá integrar el prospecto general que la Facultad se propone enviar a todos los colegas de la República en los primeros días de enero próximo, para que puedan disponer de tiempo para hacer sus arreglos y concurrir a aquellos cursos que les sean de más interés. Con tal objeto se está insistiendo con las Cátedras para que nos envien sus proyectos en el curso del presente mes. De esta manera queremos satisfacer el anhelo de muchos colegas residentes en provincias que hom manifestado su sentimiento por no haber conocido el alcance del programa por falta deinformación oportuna.

Este acercamiento con los colegas alejados de la Capital es de importancia fundamental. Ellos están más lejos de la difusión de las publicaciones médicas, cuyo costo, además, es cada vez mayor; en cambio se sienten asediados por las publicaciones de carácter comercial, no siempre seriamente garantizadas e imparciales en su información. En tal sentido se ha incorporado al programa de la Facultad, la politica de cursillos de extensión a provincias, mediante arreglos con las asociaciones médicas locales, que con tanto entusiasmo han sido recibidos y son solicitados con insistencia. La Cátedra de Tisiología y Enfermedades Respiratorias ha recorrido ya casi todo el territorio de la República en los años anteriores y el presente y su experiencia ha sido recogida para ampliar el programa a otras Cáledras. Ultimamente se realizó el cursillo sobre Ortopedia a cargo del Profesor Belisario Sánchez León, en la ciudad de Iquitos, a solicitud especial de la Asociación Médica de Loreto.

Una contribución a la relación permanente entre la Facultad y los Graduados es la difusión gratuita del Boletín de la Cátedra de Tisiología que periódicamente es enviado a todos los colegas. Además del efecto informativo en sí, en comentarios cortos, sobre las nuevas aportaciones a la etiología, patogenia, clínica y prevención de las enfer- 
medades, tal información crea un vínculo traducido en la correspondencia privada que se establece con algunos colegas después de cada edición. El Departamento tiene el propósito de extender este sistema mediante un órgano común a las demás Cátedras. La reciente adquisición de ia Facultad de un modernísimo equipo de impresiones Multilicht, que está ya en funcionamiento, hará más factible este propósito.

En otro orden de actividades, la Facultad ha auspiciado, de acuerdo con la Embajada de Italia, en el Instituto de Bioquímica a cargo del Profesor Guzmán Barrón, un cursillo sobre Métodos cromatográticos y electroforesis diciado por el Profesor Marini Bettolo, del Instituto Superior de Sanidad de Roma.

De grandes alcances es el curso que se está dictando actualmenie, sobre Estadística Médica. Este curso se dicta por gestión del Decano ante la Fundación Fulbright, la que filontrópicamente ha enviado al Profesor Louis Munan, en una nueva muestra de la benéfica política de cooperación científica de las Fundaciones norteamericanas, que la Facultad reconoce y aprecia en su gran valor. Este curso ha tenido éxito superior al esperado. Al hacer la programación, el Departamento calculó la inscripción para veinte graduados. Antes de cumplirse el plazo fijado, las solicitudes pasaban de ochenta y la insistencia de ellas obligó a solicitar del Dr. Munan el esfuerzo de realizar dos cursos paralelos, cada uno para cuarenta médicos. Así se está realizando y el interés de los concurrentes está siendo cada vez más intenso.

Se ha iniciado así el régimen de cursos a cargo de profesores visitantes, el cual será continuado. El Departamento cuspiciará en colaboración con la Sociedad Peruana de Cardiología, un curso especial a cargo del Protesor Sodi Pallares, el que se realizará en Mayo próximo.

Se están realizando conversaciones con el señor Harriman. Director del Consejo Británico para la realización de un curso de doce sesiones sobre Cirugía Toráxica, a cargo del Prolesor inglés Alphonso Abreu, quien vendría con tal objeto en el mes de enero próximo.

Para facilitar la venida de Profesores visitantes, se han iniciado conversaciones con los Departamentos de Graduados de Escuelas Médicas de otros países sudamericanos, a fin de compartir, en determinados casos los gastos de viaje de tales invitados.

Otro aspecto de las actividades del Departamento es el de procurar un mejor entendimiento en lo que se refiere al viaje al extranjero de graduados jóvenes con propósito de mejoramiento de conocimientos o de especialización. Es interés de los hospitales o entidades que 
proporcionan becas $u$ ofrecen posiciones para internos o residentes, en los Estados Unidos y algunos países europeos, recibir personal eficiente e idóneo, intelectual y moralmente, y es interés nuestro que los egresados que adquieren esos beneficios seon realmente exponentes de la mentalidad médica creada en nuestra Escuela y un índice de la capacidad de nuestra juventud profesional. La creación de la ficha del estudiante que ha hecho la Facultad por intermedio de la oficina de Estadística, creada el último año y la obtención y coordinación de datos sobre la vida del estudiante de Medicina y las actividades de los Graduados en el país, ofrece una fuente de información valiosa para el cumplimiento de esos propósitos. Son ya numerosas las conexiones que en tal sentido realiza el Departamento de Graduados y está recibiendo pedidos de información y colaboración en relación a la selección de candidatos.

El Departamento considera como tarea fundamental la de regularizar la formación de especialisias. Conforme existen tres tendencias paralelas y mutuamente integradas en la educación médica normal: la de las ciencias básicas, la de las clínicas y la de la Medicina Preventiva; así también hay tres sectores diferenciados en cuanto a la formación de especialistas. En todos los países, la formación espontánea de las especialidades se ha dirigido preferentemente al segundo grupo. La cirugía como especialidad se ha subdividido en capítulos cada vez más desarrollados, cada una de las ramas de la Clínica Médica ha buscado su autonomía. Algunas veces, tal tendencia, ha llevado al errado sistema de la especialización precoz, precisamente por la falta de un criterio orientador a la vez que coordinador en la función de la especialización. En los grandes centros de trabajo médico, la distribución de los colegas dentro de cada dedicación es bastante irregular pareciendo que hubiera exceso de un tipo de especialistas con relativa escasez en otros campos. En cambio en las poblaciones más pequeñas hay escasez o ausencia completa de profesionales a dedicación diferenciada, acorde esto con la escasez de médicos en tales lugares.

En cambio, es hecho de observación mundial, la falta de médicos dedicados a las ciencias fundamentales, como especialidad. Anatomistas, Fisiólogos y anátomo patólogos, químicos y bacteriólogos hacen falta, aun en los centros bien desarrollados. La necesidad de auxiliar 
a la clínica en pruebas de diverso orden ha creado una especialidad no bien diferenciada, de colegas dedicados a servir a la clínica en la urgencia de cada momento, empleando técnicas de laboratorio; de este modo los laboratoristas reunen en una sola dedicación, actividades fundamentalmente diferentes, cuyo único vínculo es el hecho físico de desarrollarse en un laboratorio.

Igual escasez confrontan todos los paises en cuanto se refiere a Médicos Sanitarios. Sinembargo el desarrollo de los programas de sanidad crece con igual velocidad que cambian las condiciones de vida de la población mundial. Dentro del estado actual, los médicos generales y no pocas veces, los clínicos especialistas tienen que dirigir, controlar y ejecutar aquellos programas. De otro lado, hemos visto en algún país, cómo la función sanitaria se resiente porque los médicos funcionarios olvidan sus conocimientos básicos de medicina para sobreponer postulados de Medicina Preventiva que no se coordina con la realidad. Esio hace pensar cada vez más en la necesidad de procurar que la formación de tal calidad de especialistas, se haga dentro del ambiente de una escuela médica.

En la primera etapa del programa de la Facultad para la formación de especialistas, se há pensado en los anátomo-patólogos. Además de la urgente necesidad de tenerlos, existe la circunstancia especial, entre nosotros, de existir una Cátedra, la del Profesor Pedro Weiss, con condiciones favorables, derivadas de la calidad del Jefe y de la escuela que él mismo ha formado. Además, otra muestra de la filantropia de las organizaciones norteamericanas para la protección a la Ciencia, esta vez de la Fundación Kellogg, permitirá la construcción inmediata de un edificio para el Instituto de Anatomía Patológica en terrenos del Hospital Arzobispo Loayza, cuyo uso ha sido autorizado por la Sociedad de Beneficencia Pública de Lima. En el nuevo edificio está incluído el departamento para Médicos Residentes en Patología, pero adelantándose a esa realización, el Departamento para Enseñanza de Graduados ha obtenido de la Cátedra la reglamentación respectiva, se ha realizado un concurso de selección, han sido designados tres médicos para ocupar las becas de residencia que proporciona la Facultad, se les ha proporcionado un adecuado alojamiento provisional y en ceremonia especial realizada el 15 del mes próximo pasado, se declaró inaugurado el primer programa de Residentes de la Facultad de Medicina de Lima, comenzando a ejecutarse inmediatamente el trabajo. Los Profesores a tiempo completo con quienes la Facultad ha dotado a la Cátedra, tienen función principal en el adiestramiento de los 
becarios y ellos mismos, contribuirán a la permanencia de los servicios de anatomía patológica dentro de las necesidades diarias de la función asistencial hospitalaria. El programa de adiestramiento en esta especialidad durará tres años. Cuando los que hayan cumplido satisfactoriamente el trabajo del primer año, deban seguir el segundo, ingresarán nuevos residentes al primero.

Con criterio semejante se iniciará muy en breve el programa de residentes en Radiología. La circunstancia feliz de que por gestión del Presidente de la Sociedad de Beneficencia Pública de Lima, Dr. Os. waldo Hercelles, ha sido inaugurado el Instituto Cayetano Heredia, que es uno de los mejores en su género, en América Latina, y la de que la Facultad haya podido contribuir a la obra en adecuarla mejor a los fines de la docencia; y la feliz coincidencia de que la dirección esté en manos del Profesor Titular de la Cátedra en la Facultad de Medicina, Prof. Oscar Soto hacen que el programa de Residentes pueda ser iniciado. Actualmente se está construyendo, anexo al Instituto, el Departamento que alojará a los becarios, quienes como en Patología, además de recibir el beneficio de la instrucción, cumplirán la función de permanencia durante todo el día de los servicios radiológicos para la urgencia asistencial hospitalaria.

Este programa de residencias se irá ampliando a medida de que las Cátedras estén en condiciones de realizar esta función. En los edificios para laboratorios que se construyen para el trabajo del internado, en el segundo piso, la Facultad construye también los departamenlos adecuados para iniciar las residencias en Medicina y Cirugía que comprende el programa de 1958.

En colaboración en el Departamento de Tuberculosis del Ministerio de Salud Pública se iniciará el programa de Residencia en el Hospital-Sanatorio $N^{0}$ l e igualmente en Obstetricia en la Maternidad de Lima.

La información que me he permitido presentar a Uds. en este ciclo sobre Educación Médica que ha organizado nuestra Facultad, no es simplemente la expresión de un viejo anhelo sentido y expresado reiteradamente por maestros y alumnos; es ya una realidad en marcha. Tiene los tropiezos y dificuliades detoda iniciación, pero tiene toda la seguridad de un beneficio efectivo y permanente y progresivo, la Educación Médica, en nuestra Facultad, se está desarrollando con 
un profundo y auténtico sentido de reforma. Ajeno a las veleidades de la improvisación y de corrientes noveleras, el espíritu universitario de su claustro, se robustece con la seriedad de la obra, la serenidad en el juicio y la seguridad en sus actividades. Nuestro Decano, el Profesor Alberto Huriado, está profundamente compenetrado de estos principios y su gestión, segura la mutua comprensión de los deberes y do rechos, que en sus relativas esferas, tienen todos, quienes enseñan y quienes aprenden en esta escuela, en la que venimos a prepararnos a ser médicos, esto es, protesionales responsables de la gran tarea que exige tan celosamente el ejercicio de nuestra capacidad como el de nuestro más profundo y sincero sentido humano. 